International Journal of Pure and Applied Mathematics

Volume 83 No. 1 2013, 101-110

ISSN: 1311-8080 (printed version); ISSN: 1314-3395 (on-line version)

url: http://www.ijpam.eu

doi: http://dx.doi.org/10.12732/ijpam.v83i1.9

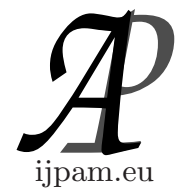

\title{
DIFFERENCE EQUATION OF LORENZ SYSTEM
}

\author{
Wanqing Song ${ }^{1 \S}$, Jianru Liang ${ }^{2}$ \\ ${ }^{1,2}$ College of Electronic and Electrical Engineering \\ Shanghai University of Engineering Science \\ Shanghai, 201620, P.R. CHINA
}

\begin{abstract}
This paper uses difference equation to explore some of the more obvious properties of the Lorenz equation. We will investigate changes in the behavior of solutions of the Lorenz equation as the parameter $r$ is varied. A trajectory in phase space is analyzed when iterative equation is magnified. The distance between two states $i$ and $j$ in the phase space is described by recurrence plots which may reveal the motion feature of chaos system trajectory with time change.
\end{abstract}

AMS Subject Classification: $65 \mathrm{P} 20$

Key Words: discrete Lorenz, phase space trajectories, recurrence plot

\section{Introduction}

The Lorenz equation is a model of thermally induced fluid convection in the atmosphere and published ${ }^{[1,2]}$ by E.N Lorenz (an atmospheric scientist) of M.I.T. in 1963. In Lorenz's mathematical model of convection, three state variables are used $(x, y, z)$. they are not spatial variables but are more abstract. The variable $x$ is proportional to the amplitude of the fluid velocity circulation in the fluid ring, positive representing clockwise and negative representing counterclockwise motion. The variable $y$ is temperature difference between up

Received: October 23, 2012

(c) 2013 Academic Publications, Ltd.

$\S_{\text {Correspondence author }}$ url: www.acadpubl.eu 
and down fluids and $\mathrm{z}$ is the distortion from linearity of the vertical temperature profile. The model is continuous in time, but a modification of the continuous equation to a discrete quadratic recurrence equation known as the Lorenz map is also widely used. The continuous version of the Lorenz model is described by the differential equation:

$$
\left\{\begin{array}{l}
x^{\prime}=a(x-y) \\
y^{\prime}=-x z+r x-y \\
z^{\prime}=x y-b z
\end{array}\right.
$$

The Lorenz system includes three equations and three parameters, three obviously property are:

(1) Nonlinearity - the two nonlinearities are $x y$ and $x z$;

(2) Symmetry - Equations are invariant under $(x ; y) \rightarrow(-x,-y)$. Hence if $(x(t) ; y(t) ; z(t))$ is a solution, so is $(-x(t) ;-y(t) ; z(t))$;

(3) Volume contraction - The Lorenz system is dissipative i.e. volumes in phase-space contract under the flow. Where the dimensionless parameters: $\mathrm{a}=$ "Prandtl number" is taken to be $10, b$ is related to the horizontal wave number of the convective motions and to be $8 / 3$. The remaining parameter $r$ is called the Rayleigh number. It is proportional to the difference in temperature from the warm base of a convection cell to the cooler top.

In 1963, illustration recreates what Lorenz described at the time. His computer calculated a trajectory using six places of accuracy, but printed the list of values using only three. When he stopped the trajectory, he used the threeplace data at the stopping time to start it up again and was puzzled when the results did not match a previous run of the trajectory that did not have a stop in the middle. That is when he concluded that the missing last three places of accuracy must be important. Lorenz observed that nonlinear systems exhibit sensitive dependence on initial conditions-very small changes in initial conditions can make very large differences in long-term behavior. the trajectory $(x(t), y(t), z(t))$, when $r=28$, approaches a strange attractor, that is, an attractor that is not an equilibrium, nor a cycle, nor a finite graph.

Because Eq. (1) is that it is non-linear, this equation has remarkable nontrivial properties and therefore it must be handled with special methods. At present, solution chaos equation mainly integrated with Runge-Kutta method of fourth-order. However, the paper will solve the Lorenz system using discrete equation, the method is convenient and intuitionistic for analyzing property of Lorenz system, and program design is also simple. 


\section{Discrete Lorenz Equation}

The approximate version of the Lorenz equation (1) is written:

$$
\left\{\begin{array}{l}
\frac{x_{k+1}-x_{k}}{d t}=a\left(x_{k}-y_{k}\right) \\
\frac{y_{k+1}-y_{k}}{d t}=-x_{k} z_{k}+r x_{k}-y_{k} \\
\frac{z_{k+1}-z_{k}}{d t}=x_{k} y_{k}-b z_{k}
\end{array}\right.
$$

The discrete or iterative version of the Lorenz model is written:

$$
\left\{\begin{array}{l}
x_{k+1}=x_{k}+a\left(x_{k}-y_{k}\right) d t \\
y_{k+1}=y_{k}+\left(r x_{k}-x_{k} z_{k}-y_{k}\right) d t \\
z_{k+1}=z_{k}+\left(x_{k} y_{k}-b z_{k}\right) d t
\end{array}\right.
$$

So for the particular parameter values have been concentrated:

$$
a=10, \quad b=\frac{8}{3}=2.666 \cdots, \quad r=28
$$

What happens if these parameters are changed? It turn out that the dependence of the solution structure on these parameters is very complicated. Depending on the choices of these parameters, you can find limit cycles, intermittent chaos, noisy periodicity, as well as strange attractors. Here we will first describe the fascinating properties of the discrete version of the Lorenz equation. In terms of equation (3), we can solve this equation with MATLAB as follow: $x(1)=1.2 ; y(1)=1.3 ; z(1)=1.6 ; \mathrm{dt}=0.005 ; a=10.0 ; r=28 ; b=2.666667$

for $k=1: 8000 x(k+1)=x(k)+a \times(y(k)-x(k)) \times \mathrm{dt}$;

$y(k+1)=y(k)+(r \times x(k)-y(k)-x(k+1) \times z(k)) \times d t z(k+1)=z(k)+(x(k) \times y(k)-$ $b \times z(k)) \times d t ;$

$x(k)=x(k+1) ; y(k)=y(k+1) ; z(k)=z(k+1) ;$

end

the program running time is long, we have to improve it follow to reduced running time:

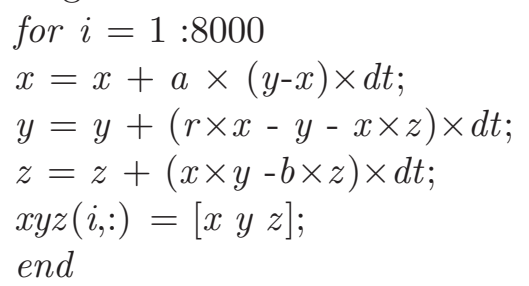

Here we will first describe the fascinating properties of the discrete version of the Lorenz equation. How to understand the behavior of the following innocentlooking difference equation? 

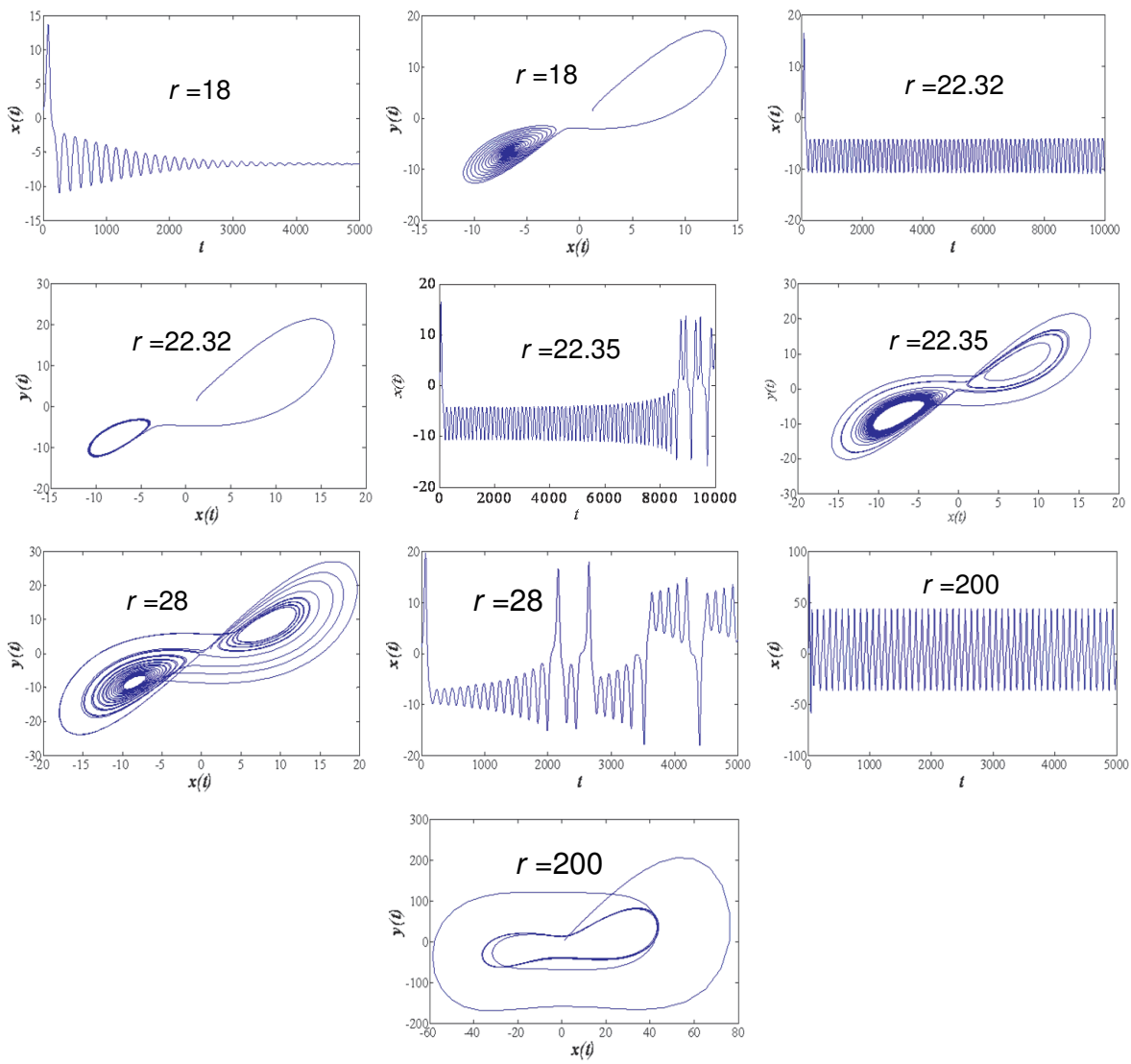

Figure 1: Phase space trajectories and waveform of time domain

Let initial value $x, y, z=(1.2,1.3,1.6)$, and run the program above. Now repeat the process for $r=18.0, r=22.32, r=22.35, r=28.0$, and $r=$ 200.0, discrete time interval $d t=0.005$. With $r$ increases some changes should be observed in phase plane and time domain as shown in Figure 1.

Chaotic behavior begins when $r=22.35$. When $r \leq 22.32$, all trajectories approaches an equilibrium. For values of $r=18$, trajectories will appear to be chaotic for an interval of time before approaching the equilibrium. This phenomenon is called transient chaos. Nonlinear systems, which exhibit chaotic behavior for certain choices of parameters, exhibit a variety of interesting behaviors, for example, when $r=200$, all trajectories approach an equilibrium again. On the $x-y$ phase plane at chaotic state, unstable spirals trajectories is 
attracted by two attractors which is called strange attractor (sometimes called the 'butterfly attractor' ). Lyapunov exponent link with self-similarity of fractal dimension $[3,4]$.

\section{Iterative Property After Discrete Equation Magnified}

The discrete or iterative version of the Lorenz equation is written:

$$
\left\{\begin{array}{l}
x_{k+1}=\left(x_{k}+a\left(x_{k}-y_{k}\right) d t\right) q \\
y_{k+1}=\left(y_{k}+\left(r x_{k}-x_{k} z_{k}-y_{k}\right) d t\right) q \\
z_{k+1}=\left(z_{k}+\left(x_{k} y_{k}-b z_{k}\right) d t\right) q
\end{array}\right.
$$

Let $a=1.0, r=28.0, b=2.666667$, original value is $x=1.2, y=1.3, z=1.6$, $d t=.005$, Lorenz system is convergence. With iterative times increasing, $q=1.001$ to 1.0048 , Lorenz system is gradually from convergence to divergence as shown in Figure 2.
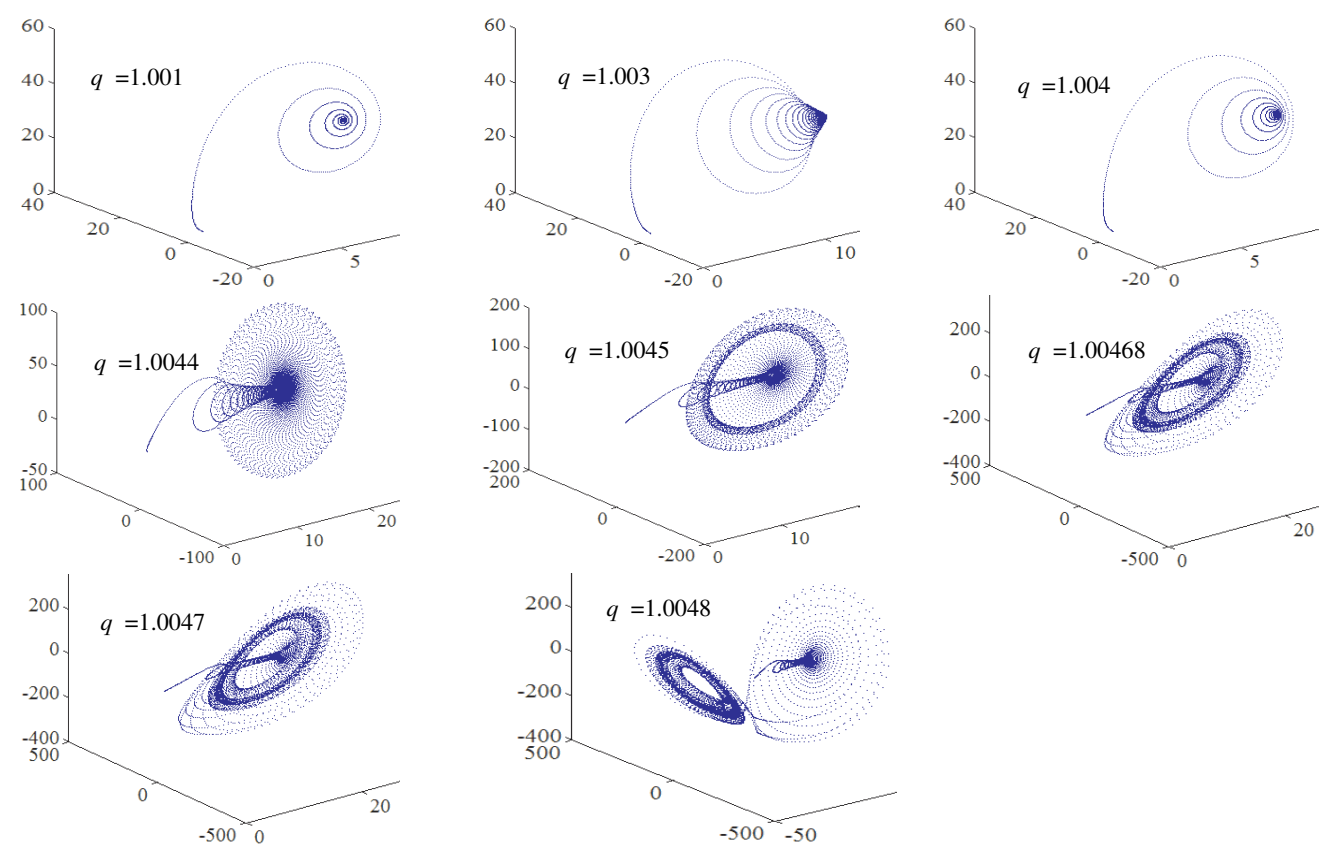

Figure 2: Phase space trajectories of 8000 point

When $a=1, r=10, b=1$, phase space trajectories in Eq. (4) show Figure 3. for $i=1: 8000$ 

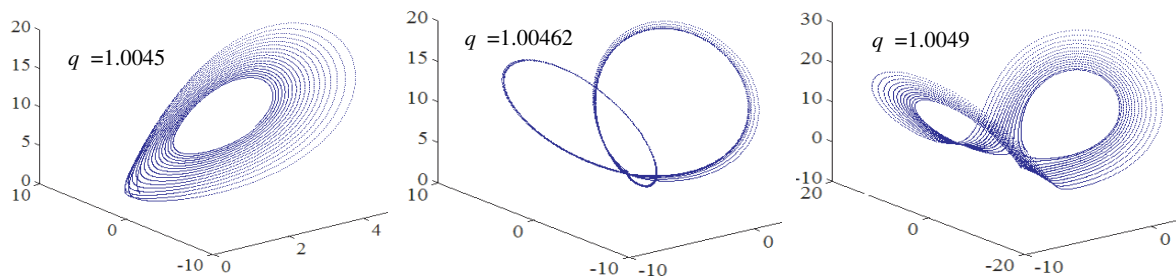

Figure 3: Phase space trajectories of 8000 point when $a=1, r=10, b=1$

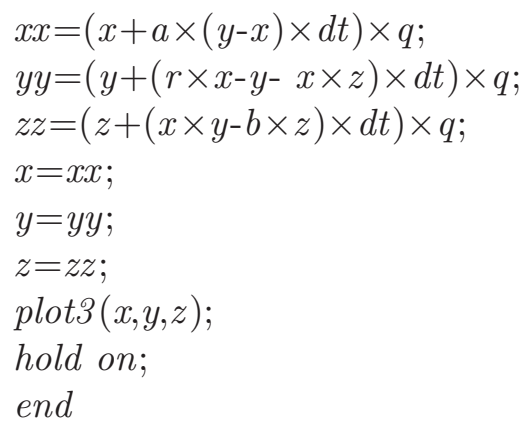

\section{Recurrence Plots of Lorenz System}

If two initial conditions have a small difference $\delta_{x}$, their difference after time $\mathrm{t}$ will be $\delta_{x} e^{\lambda t}$ with $\lambda>0$, that is, exponential separation. Thus, a tiny difference or even a cutoff error will be blown up quickly and results in a big difference in the near future. "Similar causes have similar effects" is invalid in chaotic systems except for short periods, as shown in Figure 4. A system is called chaotic system, provided that its maximum Lyapunov exponent is positive $[5,6,7]$

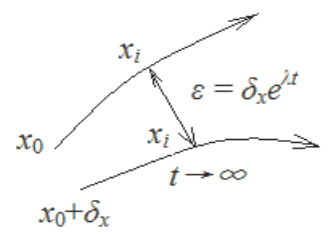

Figure 4: $x(t)$ chaotic trajectories when initial conditions has a small difference $\delta_{x}$

The phase space trajectories of chaos equations that have more than three dimensions are difficult to visualize. Recurrence plots ${ }^{[8]}$ can well solve the 
problem for analyzing higher dimensional system. to detect transitions between different chaos system or to find interrelations between several systems. The recurrence plot may visualize the recurrences of any one variable state in the phase space by a two-dimensional plot of time domain.

\subsection{Definition of Recurrence Plots}

Let $x(i)$ be the $i$-th point on the orbit describing a Lorenz equation in $d$ dimensional space, for $i=1, \ldots, N$. The recurrence plot is an array of dots in a $N \times N$ matrix, where a dot is placed at $(i, j)$ whenever $x(j)$ is sufficiently close to $x(i)$. In practice one proceeds as follows to obtain a recurrence plot from a time series $u_{i}$. First, choosing an embedding dimension $\mathrm{m}$, one constructs the m-dimensional orbit of $x(i)$ by the method of time delays $\tau$, then

$$
x(i)=\left(u_{i}, u_{i+\tau}, \ldots, u_{i+(m+1) \tau}\right)
$$

Next, one chooses $\varepsilon(i)$ such that the ball of radius $\varepsilon(i)$ (centered at $x(i)$ in $\boldsymbol{R}^{\mathrm{m}}$ contains a reasonable number of other points $x(j)$ of the orbit. In our computation we have allowed the radius $\varepsilon(i)$ to depend on the point $x(i)$, and $\varepsilon(i)$ has been selected by a routine used in our algorithm for the determination of the Lyapunov exponents. Finally, one plots a dot at each point $(i, j)$ for which $x(j)$ is in the ball of radius $\varepsilon(i)$ centered at $x(i)$. We call this picture a recurrence plot.since the $i, j$ are in fact times, a recurrence plot describes natural (but subtle) time correlation information. Recurrence plots tend to be fairly symmetric with respect to the diagonal $i=j$ because if $x(i)$ is close to $x(j)$, then $x(j)$ is close to $x(i)$. There is, however, no complete symmetry because we do not require $\varepsilon(i)=\varepsilon(j)$.

\subsection{Constructing Recurrence Plots of a Variable $x(t)$ in the Lorenz Equation}

If the distance between two states $i$ and $j$ on the variable $x(t)$ trajectory is smaller than a given threshold $\varepsilon$ the value of the recurrence matrix $R$ is one, otherwise zero ${ }^{[9]}$, see Eq. (5). The recurrence plot is then the twodimension display of the $N \times N$ matrix, where black pixels represent $R_{i, j}=1$ and white pixels indicate $R_{i, j}=0$. Such recurrence plot can help to find a first characterization of the Lorenz system of data or to find transitions and interrelations of the system.

$$
R_{i, j}=\left\{\begin{aligned}
0, & \left\|x_{i}-x_{j}\right\| & >\varepsilon \\
1, & \left\|x_{i}-x_{j}\right\| & \leq \varepsilon
\end{aligned}\right.
$$


In order to plot the distance matrix, let a threshold $\delta=5$, delay time $\tau=2$, embedding dimension $m=3$. For a variable $x(t)$, we need to transfer the trajectory vector $x(i)$ and $x(j)$ into test vectors, whose component wise test will provide the pair-wise test of the trajectory vector. Using the two vectors we calculate the recurrence plot using plot the Euclidean norm in Eq. (5) and a coordinate system representing two time axes. The distance matrix generates the black/while recurrence plot, as shown in Figure 5. The pixels regions correspond to sequences $(i, j),(i+1, j+1), \ldots,(i+k, j+k)$ such that the piece of trajectory $x(j), x(j+1), \ldots, x(j+k)$, is close to $x(i)$, $x(i+1), \ldots, x(i+k)$.

Because a recurrence plot describes the time correlation information, for $r=22.32$ and $r=200$, the regions in Figure 5 is visible texture in the form of short lines (like points) parallel to the diagonal of the recurrence plot, it means the variable $x(t)$ take on a periodic state. For $r=28$, the generated checkerboard texture corresponds to the fact that $x(i)$ moves on a chaotic states. The length of the lines is thus related to the inverse of the largest positive Lyapunov exponent.

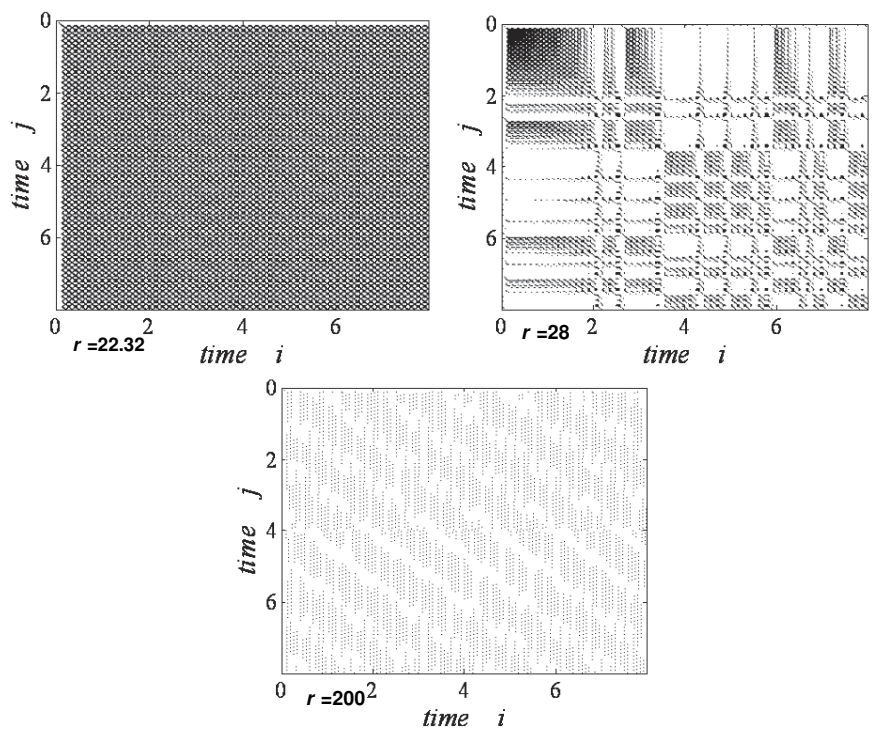

Figure 5: The recurrence plot of the variable $x(t)$, the time is $8 \mathrm{~s}$ with $\varepsilon=5, \tau=2, m=3$

A careful analysis shows that the diagonal black spots fall into two mutually exclusive groups having again black spots on the intersection of the horizontal 
and vertical lines. The distances between these diagonal lines, representing the periods of the cycles, differ and not constant-just as they are in a chaotic states.

\section{Conclusion}

Lorenz equation and its variants have been well studied in the past decades. A lot of important results have been achieved. This paper is contributed to the brief summary of the discrete Lorenz equation with Matlab. When the discrete Lorenz equation is enlarged $q$ times $(q>1)$, the property of an iteration process been discussed. The recurrence plots of the Lorenz are rather easily obtained aids for the diagnosis of dynamical systems. They display important and easily interpretable information about time scales which are otherwise rather inaccessible. Bivariate and multivariate extensions of recurrence plots furthermore offer nonlinear correlation tests and synchronization analysis. In the paper mentioned fields, new techniques and methods are continuously explored.

\section{References}

[1] Francis C. Moon, Chaotic and Fractal Dynamics, Wiley-Interscience Publication, New York (2008).

[2] Chaos Suppression of Generalized Lorenz System: Adaptive Fuzzy Sliding Mode Control Approach, 2008 IEEE Conference on Soft Computing in Industrial Applications (SMCia/08), June 25-27, 2008, Muroran, Japan.

[3] Michael F. Barnsley, Fractals Everywhere, Second Edition, Elsevier, Singapore (2009).

[4] Zonghua Liu, Chaotic Time Series Analysis, Mathematical Problems in Engineering (2010).

[5] Qais H. Alsafasfeh, Mohammad S. Al-Arni, New chaotic behavior from Lorenz and Rossler systems and its electronic circuit implementation, Circuits and Systems, 2 (2011), 101-105.

[6] Alaa M. Al-Roumy, The study of a new Lorenz-like model, Journal of Basrah Researches (Sciences), 37, No. 3A (2011).

[7] M. Moghtadaei, M.R. Hashemi Golpayegani, Complex dynamic behaviors of the complex Lorenz system, Scientia Iranica (2011). 
[8] J.P. Eckmann, S.O. Kamphorst, D. Ruelle, Recurrence plots of dynamical system, Europhsics Letters, 5 (1987), 973-977.

[9] Martin H. Trauth, Matlab Recipes for Earth Sciences, Second Edition, Springer Berlin Heidelberg (2007). 